\title{
Interactivity for Museums: Designing and Comparing Sensor-Based Installations
}

\author{
Pedro Campos ${ }^{1}$, André Dória ${ }^{1}$, and Magno Sousa ${ }^{2}$ \\ ${ }^{1}$ University of Madeira \\ Campus Universitário da Penteada, 9000-390 Funchal, Portugal \\ ${ }^{2}$ WowSystems \\ R. da Alfândega, 78, 3ㅇ, 9000-059 Funchal, Portugal \\ pcampos@uma.pt, andredoria@netmadeira.com, \\ magno. sousa@wowsystems.pt
}

\begin{abstract}
Technology today provides exciting new possibilities for creating more appealing museum experiences, since we can exploit the surprise factor of innovative interaction styles to create an engaging experience that facilitates the learning process. This research was based on extensive in-site observation as well as surveys conducted at the scene, and it relates to a cultural interactive exhibition titled "Cultural Tourism". The issues were organized around usability problems detected, social interaction and differences between interaction styles, all of which contribute to increasing our knowledge regarding the use of interactive technology as a means to reduce the distance between visitors and cultural heritage.
\end{abstract}

Keywords: Interactive installations, museum exhibitions, sensor-based installations, kiosks, field observations.

\section{Introduction}

The use of sensor-based interactive installations, in particular installations involving infrared motion sensors as well as cameras coupled with real time video processing algorithms, have been receiving considerable interest both from industry and academia $[1,2,3]$. During the design and evaluation of interactive exhibitions, much can be learned about interaction design for public settings like these.

We describe our own experience designing and evaluating an interactive exhibition, which featured four different interaction styles to control digital contents: touching, walking over, waving and page-flipping. Our design approach was tailored to the exhibition's contents and makes a creative use of sensor-based technology, with the explicit goal of reducing the distance between visitors and cultural heritage [7].

The remainder of this paper is organized as follows: Section 2 describes related work with a particular emphasis on adding interactivity to cultural heritage exhibitions and museums. Section 3 describes the interactive installations designed as well as the interaction styles employed. Finally, Section 4 described the evaluation approach and results, drawing some conclusions organized around usability problems detected, social interaction among visitors and differences between interaction styles. 


\section{Related Work}

Technology today provides exciting new possibilities to approach museum visitors to culture and heritage. Danks and colleagues [1] refer the focus shift towards using interactive artifacts to enhance the visiting experience, which contrasts with the traditional approach, centered on the museum's collection, display and storage of objects. They argue that today's museum visitor expects to learn while also having fun at the same time, therefore interactive storytelling and gaming have a great potential to improve modern museum's experiences [1].

Several experiences have been conducted to study how visitors experience novel interaction styles within museums and science centers. Explore@Bristol, for instance, was an interactive science museum, which was studied to analyze six of its exhibits according to three dimensions: Drama/Sensation, Challenge/Self expression and Social [5]. A "Walk in the Wired Woods" illustrates how to design an engaging experience through context-sensitive media and interaction. The visitors were invited to take a walk in which they were automatically presented with audio content appropriate to their physical location [6]. Other interesting studies have been performed, regarding novel interaction styles and schemes, applied both to leisure and educational activities. Such examples include "The Fire and the Mountain" exhibition, held in 2006 at the Civic Museum of Como, Italy [4] and the "Listen Reader" from Xerox PARC, an innovative and engaging reading experience installed in three different museums over a six-month exhibition period [3].

When studying visitors' interaction with museum digital installations, the problem of evaluation arises inevitably, since it's neither easy nor clear to establish the right set of methods in order to draw credible conclusions. Hornecker and Stifter [2], reporting on the evaluation of a digitally augmented exhibition on the history of modern media, based their conclusions on (i) logfiles' analysis, (ii) interviews and (iii) observation in the museum. We also followed this approach as a means to obtain a better understanding of the interactive installations, described in the next section.

\section{The "Cultural Tourism" Exhibition}

In April 2008, we designed a set of sensor-based installations in a cultural exhibition organized by the Direction of Cultural Affairs, which aimed at showing the visitor the cultural richness that formed the streets of Funchal (Portugal). The concepts of the exhibition revolved around promoting awareness about, and fostering a better understanding of, the cultural tourism that can be performed by simply walking through strategic streets and watching certain buildings, sites, and heritage. To better complement the exhibition's traditional large-format printed panels, the organizers wanted to have the interactivity factor as a means to add value to the visitor's experience.

The final set of installations included: (i) a virtual book that could be browsed by simple page-flipping gestures performed in mid-air; (ii) an interactive floor that illustrated the evolution of the transportation means along the years; (iii) an interactive timeline using a touch-screen and (iv) a panel with projected images that would change through waving. These installations are shown in Fig. 1. 

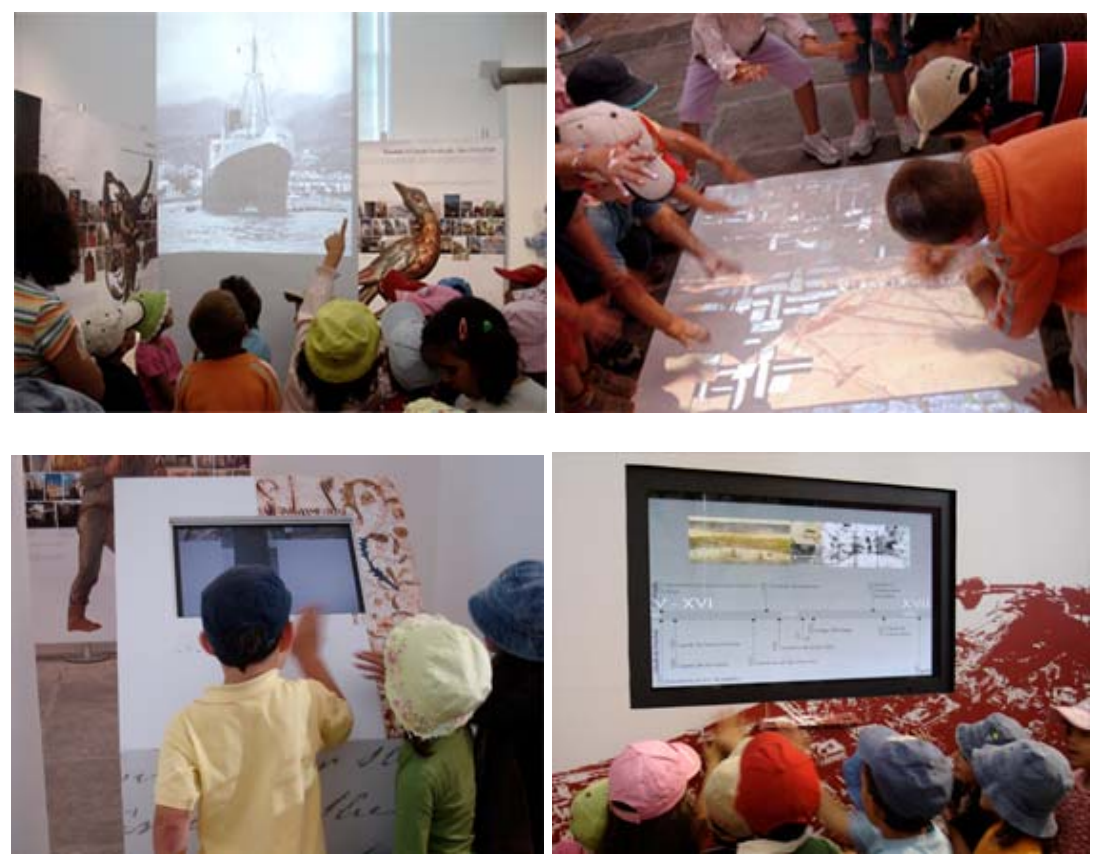

Fig. 1. The installations and interaction styles employed throughout the exhibition (clockwise from top left): waving, walking over, page-flipping and touching

\section{Results and Future Work}

The evaluation of the exhibition was conducted during 30 days, from April to May (2008), and consisted in observing visitors interacting with the installations.

The interactions were observed both in presence and video-recorded. A subset of 74 out of more than 1000 visitors was also interviewed during a debriefing session after their visit. Of these 74 visitors, 32 were under 12 years old, 21 were above 60 years old and 51 were female visitors. Additionally, qualitative data analysis was applied to all data (observer notes, video recordings and survey results) in order to gain more insight regarding the visitors' experience. This insight was organized in three different issues: usability problems found, social interaction among visitors, differences between interaction styles. We found differences in all these issues to be correlated to age, e.g. younger visitors exhibited a much larger degree of social interaction than older visitors, and they also enjoyed one particular interaction style that wasn't as much appreciated by older visitors: walking over the interactive floor.

In general, visitors were very pleased with the interactive installations. In the debriefing interviews, we asked them to rate some aspects using a 5-point Likert scale:

- How much they enjoyed the exhibition -4.7

- Interaction style rating: walking (4.8), page-flipping (4.2), touching (3.9) and waving (3.7). (all values are an average over 74 answers) 
Usability problems found. In general, children had no difficulties interacting with the installations, even though they most of them had never used interactive installations of the kind. Older visitors were more averse to interacting and preferred to simply watch. Some visitors didn't immediately realize the installations were interactive: people are not used to this kind of exhibitions. Affordances are needed so that visitors know they can interact with the installations: this would have grabbed much more attention and visitors.

Social interaction. As we mentioned, there were much larger levels of social interaction among children than adults. But the most successful ones were clearly the pageflipping gestures performed at the virtual book and the interactive floor. The interactive floor, in particular, allowed collaborations between visitors that wanted to discover the images underneath them and sparked conversations (about culture) between visitors who interacted with the floor at the same time.

Differences between interaction styles. We observed that there was a tendency to "transport" interaction styles from one installation to the next, i.e. visitors trying to "page-flip" a touch-screen. The collaboration allowed by the interactive floor clearly enhanced the level of engagement and their focus. Regarding the virtual book, it was interesting to note the important role of affordances, which were adequate to the interaction style: you browse the virtual book the same way you browse a real book.

One of the main problems we face today, when trying to provide museum experiences incorporating interactive technologies, is to find out issues that could guide future designs: in this paper, we learned, for instance, the importance of showing visitors they can interact with screens and floors, since people aren't still used to this kind of interactive technologies. What the HCI community needs are more concrete design case studies coupled with observational analysis drawn from those case studies. In this sense, we believe our contribution is a first step towards bringing people closer to their cultural heritage.

\section{References}

1. Danks, M., Goodchild, M., Rodriguez-Echavarria, K., Arnold, D.B., Griffiths, R.N.: Interactive storytelling and gaming environments for museums: The interactive storytelling exhibition project. In: Hui, K.-c., Pan, Z., Chung, R.C.-k., Wang, C.C.L., Jin, X., Göbel, S., Li, E.C.-L. (eds.) EDUTAINMENT 2007. LNCS, vol. 4469, pp. 104-115. Springer, Heidelberg (2007)

2. Hornecker, E., Stifter, M.: Learning from interactive museum installations about interaction design for public settings. In: Kjeldskov, J., Paay, J. (eds.) Proceedings of the 18th Australia Conference on Computer-Human interaction. OZCHI 2006, vol. 206. ACM, New York (2006)

3. Back, M., Cohen, J., Gold, R., Harrison, S., Minneman, S.: Listen reader: an electronically augmented paper-based book. In: Proceedings of the SIGCHI conference on Human factors in computing systems, pp. 23-29. ACM Press, New York (2001)

4. Garzotto, F., Rizzo, F.: Interaction paradigms in technology-enhanced social spaces: a case study in museums. In: Proc. DPPI, pp. 343-356. ACM Press, New York (2007)

5. Kidd, A.: Technology experiences: What makes them compelling? Hewlett Packard Laboratories, Bristol, UK (2001)

6. Hull, R., Reid, J., Geelhoed, E.: Social impacts of computing: Codes of professional ethics. Pervasive Computing 1(4), 56-61 (2002)

7. Dória, A., Campos, P., Fernandes, E.: Designing an Interactive Forest through Sensor-based Installations. In: Proceedings of CHI 2008, Florence, Italy (2008) 